\title{
A DESCRIÇÃO MATEMÁTICA DO FENÔMENO OSCILATÓRIO DU- RANTE A SÍNTESE ELETROQUÍMICA DOS FILMES DOS OXI-HIDROXI COMPOSTOS DE COBALTO (III) E DURANTE A SÍNTESE DOS COM- PÓSITOS DELES COM OS POLÍMEROS CONDUTORES
}

\author{
V.Tkach*, V.Nechyporuk e O.Slipenyuk \\ Universidade Nacional de Chernivtsi, 58012, Chernivtsi, Rua Kotsyubyns'koho, 2, Ucrânia \\ *E-mail do contato volodya@1lanera.com
}

O comportamento eletroquímico do sistema da eletrotransformação potenciostática dos sais de cobalto (II) para os oxi-hidroxicompostos de cobalto (III) na presença dos íons de fluoro (que promove a dissolução do oxi-hidroxicompostos de cobalto (III)) sobre plátina, que age como oscilador, foi descrito matematicamente. O modelo matemático correspondente foi analizado através da teoria da estabilidade linear e a análise de bifurcações. É mostrado que as oscilações neste sistema ocorrem por causa da interação entre as partículas do filme.

Para o caso da eletrosíntese dos compósitos poliméricos dos oxi-hidroxicompostos de cobalto com os polímeros condutores na ausência dos íons de fluoro o fator mais responsável pelo comportamento oscilatótio é o fator da polimerização.

Palavras-chaves. Cobalto, filmes passivos, revestimentos protetores, compósitos polimericos, polímeros condutores, oscilações eletroquímicas.

\section{INTRODUÇÃO}

Cobalto é um dos metais mais usados. Os seus compostos têm muitas aplicações. O uso dos filmes dos compostos oxigênicos do cobalto como revestimento de corrosão é uma das aplicações potenciáis dos compostos dele. Os oxi-hidroxi compostos de cobalto podem-se usar também nas investigações óticas já que eles têm a cor intensamente preta.

Os autores dos trabalhos [1] e [2] conseguiram sintetizar eletroquimicamente no modo potenciostático os oxi-hidroxi compostos do cobalto no modo potenciostático sobre plátina através da oxidação dos sais do cobalto bivalente a partir das soluções que continham fluoreto. Durante o processo da síntese do composto do cobalto (III), observaram-se as oscilações em corrente. $\mathrm{O}$ experimento foi reproduzido 3 vezes com as concentrações diferentes do fluoreto de amônio. A freqüência, a amplitude e o periodo das oscilações dependia da concentração do fluoreto, como se pode mostrar na esquema.

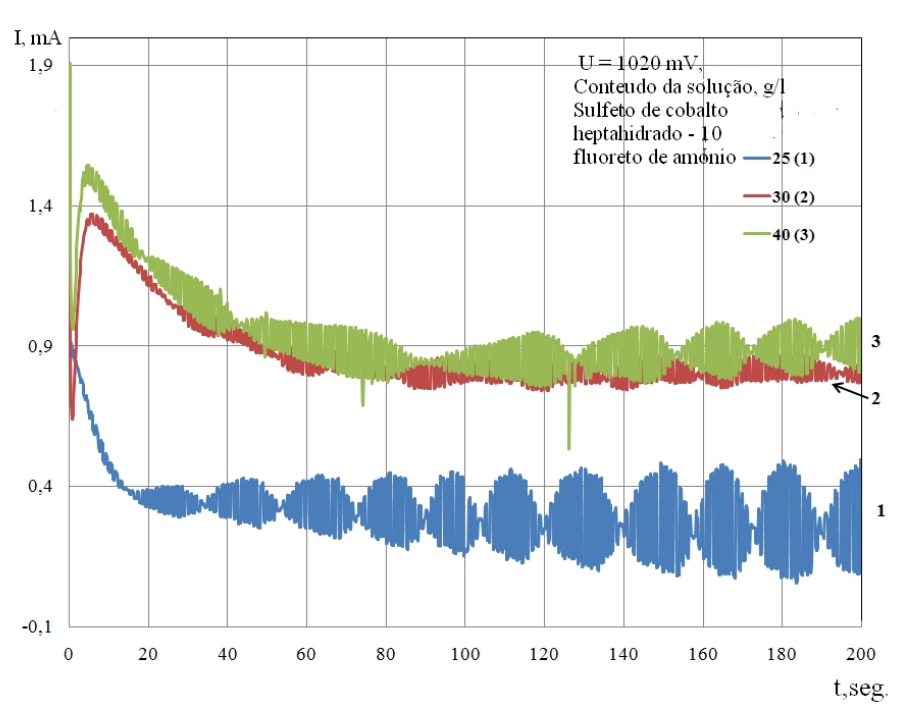

Esq. 1. As oscilações de corrente, observadas durante a oxidação eletroquímica dos sais de cobalto bivalente (para este caso do sulfato de cobalto heptahidrado) e a dependência da imágem delas da concentração do fluoreto na solução.

Os valores da corrente e da freqüência das oscilações cresceram com o aumento da concentração do fluoreto, já que a velocidade da dissolução do filme cresceu.

Quando os experimento foi repetido sob os potencias mais altos, a quantidade das oscilações se 
diminuíu, já que a aumenção do potencial promoveu a formação do filme e a razão entre as velocidades da formação e da dissolução do filme se diminuíu (Esq. 2).

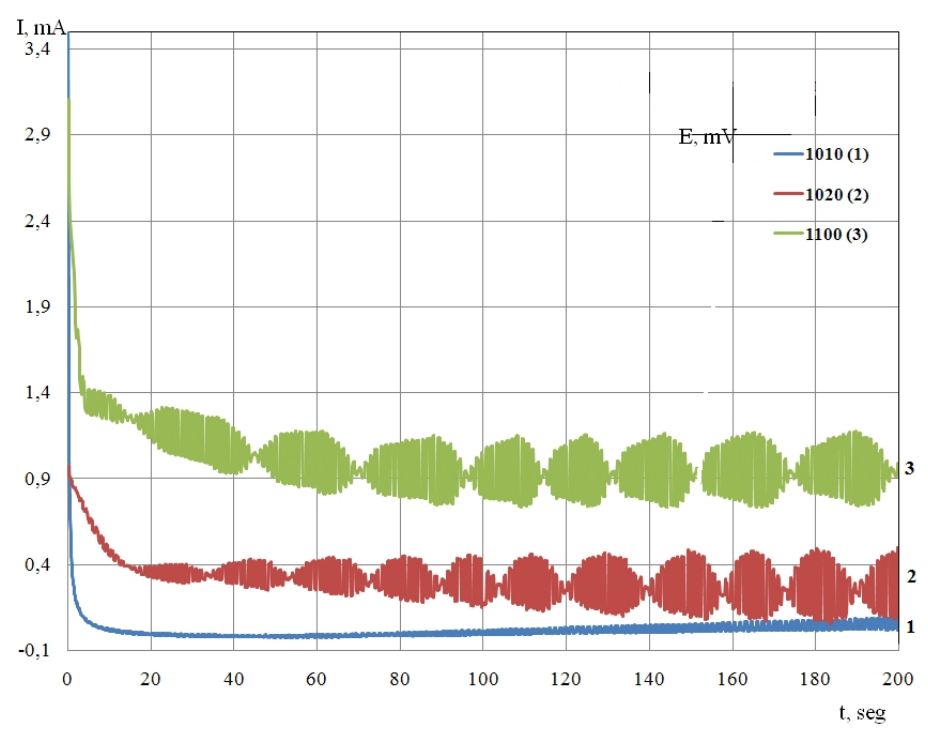

Esq. 2. As oscilações de corrente, observadas durante a oxidação eletroquímica dos sais de cobalto bivalente no modo potenciostático sob potenciais diferentes e a dependência da imágem delas do potencial.

O comportamento oscilatório não ocorreu quando o potencial do anodo foi mantido maior que $1100 \mathrm{mV}$, o estado estacionário, no qual sistema existiu, foi estável e a eletrólise conformou às equações de Tafel [2], já que a velocidade da formação e dissolução do filme foi igual (a razão entre eles foi igual a um).

Para aprofundar o nosso conhecimento do processo oscilatório neste sistema, nós temos que descrever o comportamento eletroquímico neste sistema matematicamente e analizar o modelo matemático dele usando a teoria da instabilidade linear e a análise de bifurcações. Isso dar-nos-á a possibilidade de conhecer com mais certeza o mecanismo da aparecida delas. O modelo matemático deste processo será descrito na seção primeira deste artigo.

A seção segunda deste artigo contém a descrição matemática da síntese eletroquímica do compósito dos oxi-hidroxi compostos de cobalto (III) com os polímeros condutores, cujos compósitos com outros compostos oxidos dos metais já se usam [3] a partir das soluções que não contêm fluoreto (caso contrário, o rendimento do compósito será diminuído tanto por causa da dissolução do oxi-hidroxicomposto, quanto por causa da morfologia não satisfatória do PC dopado com fluoreto [4]). O compósito $\mathrm{CP}-\mathrm{CoO}(\mathrm{OH})$ pode potencialmente ser usado nos aparelhos óticos e também como o revestimento de corrosão.

\section{A DESCRIÇÃO MATEMÁTICA DA SÍNTESE ELETROQUÍMICA DOS OXI- HIDROXICOMPOSTOS DE COBALTO (III) NAS SOLUÇÕES QUE CONTÊM FLUORETO.}

Para descrever matematicamente o comportamento eletroquímico deste sistema, nós introduzimos três variáveis

C - a concentração dos íons de cobalto bivalente na camada pre-superficial

$\Theta$ - o grau do recobrimento da superfície do eletrodo com a filme do oxi-hidroxicomposto de cobalto,

f - a concentração dos íons do fluoreto na camada pre-superficial.

Para simplificar o modelo matemático deste processo suponhamos que o liquido se está mexendo intensamente (pra menosprezar a influência do fluxo da convecção), o eletrolito de suporte está en excesso, pois podemos menosprezar a influência do fluxo da migração. A distribuição concentracional na camada da difusão é suposta a ser lineal e a expessura da camada constante e igual a $\delta$.

Os cátions de cobalto entram na camada pre-superficial através da difusão e desaparecem oxidando-se. A equação de balanço então será descrita como

$$
\frac{d C}{d t}=\frac{2}{\delta}\left(\frac{D}{\delta}(c-C)-v_{o x}\right) \equiv F_{1}
$$

Sendo c a concentração dos íons de cobalto no interior da solução, $\mathrm{v}_{\mathrm{ox}}$ a velocidade da oxidação eletroquímica dos íons de cobalto que leva à formação do filme. Ela pode-se calcular como

$$
v_{o x}=k_{o x} C(1-\Theta) \exp \left(\alpha_{1} \Theta\right) \exp \left(-\frac{z F}{R T} \phi_{0}\right)
$$

Sendo $\mathrm{k}_{\mathrm{ox}}$ a constante da oxidação, $\alpha_{1}$ o coeficiente que descreve a interação entre as moléculas do filme, $\mathrm{z}$ a quantidade de eletrons transferidos, $\mathrm{F}$ a constante de Faraday, $\mathrm{R}$ a constante universal de gases, $\mathrm{T}$ a temperatura absoluta, $\varphi_{0}$ o salto do potencial na camada 
dobra relativamente ao potencial da carga zero.

O filme na superfície. O filme se está formando a partir dos ions de cobalto através da oxidação anôdica deles. O filme desaparece da superfície dissolvendo-se reagindo com os ligandes - ánions de fluoreto. A equação de balanço então se poderá descrever como

$$
\frac{d \Theta}{d}=\frac{1}{G_{\max , f}}\left(v_{\star}-v_{d}\right) \equiv F_{2}
$$

Sendo $G_{\max }$ a concentração superficial máxima do composto na superfície, $\mathrm{v}_{\mathrm{d}}$ a velocidade da dissolução do filme, calculada como

$$
v_{d}=k_{d} F \Theta \exp \left(-\alpha_{2} \Theta\right)
$$

Sendo $\mathrm{k}_{\mathrm{d}}$ a constante da dissolução, $\alpha_{2}$ a constante que descreve a interação entre as moléculas do filme.

Os ánions de fluoreto. Chegam para a camada pre-superficial através da difusão e desaparecem reagindo com o filme dissolvendo-o. A equação de balanço deles então será descrita como

$$
\frac{d F}{d t}=\frac{2}{\delta}\left(\frac{\Delta}{\delta}(\phi-F)-v_{d}\right) \equiv F_{3}
$$

Sendo $\Delta$ o coeficiente da difusão dos íons do fluoreto, $\varphi$ a concentração deles no interior da solução.

A carga do eletrodo pode-se calcular como

$$
\mathrm{Q}=\mathrm{K}_{1}\left(\varphi_{0}-\varphi_{1}\right) \Theta+\mathrm{K}_{0} \varphi_{0}(1-\Theta)
$$

Sendo $\mathrm{K}_{0}$ e $\mathrm{K}_{1}$ as capacidades das partes da camada dobra que correspondem à parte livre e ocupada da superfície, $\varphi_{1}$ é o salto do potencial relativamente ao potencial da carga zero que se refere à parte da camada dobra que corresponde à parte ocupada da superfície do eletrodo. Então, o salto do potencial depende do grau do cobrimento da superfície e o derivativo da função que descreve essa dependência pode ser descrito como

$$
\frac{\partial \phi_{0}}{\partial \Theta}=\frac{\phi_{0}\left(\mathrm{~K}_{0}-\mathrm{K}_{1}\right)+K_{1} \phi_{1}}{\mathrm{~K}_{1} \Theta+\mathrm{K}_{0}(1-\Theta)}
$$

\section{RESULTADOS E DISCUSSÃO (SEÇÃO I)}

Para investigar o comportamento deste sistema, nós precisamos de analizar o sistema de equações (1), (3) e (5) através da teoria da estabilidade linear. Os elementos da matriz funcional de Jacobi para este sistema podem-se descrever como

$$
\begin{gathered}
a_{11}=\frac{2}{\delta}\left(-\frac{D}{\delta}-\frac{v_{o x}}{C}\right) \\
a_{12}=\frac{2}{\delta}\left(\frac{v_{o x}}{1-\Theta}-\alpha_{1} v_{o x}+\frac{z F}{R T} v_{o x} \frac{\phi_{0}\left(\mathrm{~K}_{0}-\mathrm{K}_{1}\right)+K_{1} \phi_{1}}{\mathrm{~K}_{1} \Theta+\mathrm{K}_{0}(1-\Theta)}\right) \\
a_{13}=0 ; \mid \quad a_{21}=\frac{1}{G_{\max }} \frac{v_{o x}}{C} \\
a_{22}=\frac{1}{G_{\max }}\left(\alpha_{1} v_{o x}-\frac{v_{o x}}{1-\Theta}-\frac{z F}{R T} v_{o x} \frac{\phi_{0}\left(\mathrm{~K}_{0}-\mathrm{K}_{1}\right)+K_{1} \phi_{1}}{\mathrm{~K}_{1} \Theta+\mathrm{K}_{0}(1-\Theta)}+\alpha_{2} v_{d}-\frac{v_{d}}{\Theta}\right)
\end{gathered}
$$

Para determinar as condições do estado estacionário estável, vamos usar o critério de Rauss e Gurwitz. A equação caraterística do sistema de equações (13) pode ser descrita como

$$
\Phi^{3}+\mathrm{A} \Phi^{2}+\mathrm{B} \Phi+\Gamma=0
$$

Sendo que

$$
\begin{gathered}
A=-\left(a_{11}+a_{2}+a_{3}\right) \\
B=\left|\begin{array}{ll}
a_{11} & a_{1} \\
a_{2} & a_{2}
\end{array}\right|+\left|\begin{array}{ll}
a_{1} & a_{3} \\
a_{3} & a_{3}
\end{array}\right|+\left|\begin{array}{ll}
a_{2} & a_{3} \\
a_{3} & a_{3}
\end{array}\right| \\
\Gamma=-\left|\begin{array}{lll}
a_{11} & a_{12} & a_{\mathrm{B}} \\
a_{2} & a_{2} & a_{3} \\
a_{3} & a_{3} & a_{3}
\end{array}\right|
\end{gathered}
$$

O critério de Rauss e Gurwitz requer que para um estado estacionário estável os minores dos membros da diagonal principal da matriz de Gurwitz

$$
\left(\begin{array}{lll}
A & 1 & 0 \\
\Gamma & B & A \\
0 & 0 & \Gamma
\end{array}\right)
$$


sejam positivos. Os menores dos membros da diagonal principal da matriz:

$$
\Delta_{1}=A, \Delta_{2}=\left|\begin{array}{ll}
A & 1 \\
\Gamma & B
\end{array}\right|, \Delta_{3}=\left|\begin{array}{ccc}
A & 1 & 0 \\
\Gamma & B & A \\
0 & 0 & \Gamma
\end{array}\right|
$$

Visto que $\Delta_{3}=\Gamma \Delta_{2}$, podemos formar a condição do estado estacionário estável como $\Gamma>0$. Vamos achar as condições do estado estacionário estável. Vamos apresentar as novas variáveis.

$$
\begin{gathered}
\frac{v_{o x}}{C}=s ; \frac{v_{o x}}{1-\Theta}-\alpha_{1} v_{o x}=u \\
\frac{z F}{R T} v_{o x} \frac{\phi_{0}\left(\mathrm{~K}_{0}-\mathrm{K}_{1}\right)+K_{1} \phi_{1}}{\mathrm{~K}_{1} \Theta+\mathrm{K}_{0}(1-\Theta)}=W_{1} \phi_{0}+W_{2} ; \frac{v_{d}}{F}=X
\end{gathered}
$$

O jacobiano então será apresentado como

$$
\operatorname{DetJ}=\frac{2 D}{\delta^{2} G_{\max }}\left|\begin{array}{ccc}
-s-\frac{D}{\delta} & u+W_{1} \phi_{0}+W_{2} & 0 \\
s & -u-W_{1} \phi_{0}-W_{2}+\alpha_{2} X F-\frac{X F}{\Theta} & -\mathrm{X} \\
0 & \alpha_{2} X F-\frac{X F}{\Theta} & -\mathrm{X}-\frac{\Delta}{\delta}
\end{array}\right|
$$

Os cálculos dar-nos-ão a expressão do Jacobiano, apresentada na forma simplificada. Usando a condição $\Gamma>0$ e resolvendo a inequação relativamente ao $\varphi_{0}$, poderemos obter o valor crítico do salto do potencial e desde então a condição do estado estacionário estável poderá se descrever.

$$
\phi_{0}<\frac{D(X-1)\left(u+W_{2}\right)-S X F\left(a_{2}+1\right)+X F D\left(a_{2}-1\right)}{W_{1}(D X-\Delta D)}
$$

Os autores disseram que o sistema estava no estado estacionário estável, em que o controle da oxidação foi melhor e a eletrólise conformava à equação de Tafel após aplicar ao anodo o potencial maior que $1100 \mathrm{mV}$. Segundo este modelo, a explicação é assim. Quando ao anodo foi aplicado o potencial superior ao valor mencionado o salto do potencial na camada dobrada começou a conformar à condição (11). Quando os potenciais inferiores foram aplicados ao anodo, o estado estacionário não foi estável e o sistema agia como oscilador. O valor crítico do salto do potencial depende mais da concentração dos íons de fluoro (F), diffusão dos íons de cobalto (D) e fluoro $(\Delta)$ e os efeitos da oxidação anôdica à camada dobrada $\left(\mathrm{W}_{1}\right.$ e $\left.\mathrm{W}_{2}\right)$. Também pode-se mostrar que se a interação entre as moléculas do filme (u e $\alpha_{2}$ ) for repelente (eles tiverem valor negativo), o estado estacionário também será estável).

A instabilidade oscilatória. Ocorrem nas condições da bifurcação de Hopf. Para saber as condições certas da ocorrência dela, é preciso de resolver a equação.

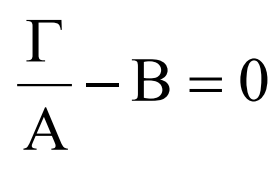

(12)

Com a condição adicional de $\mathrm{B}>0$. Para que ela se realize, é preciso (mas não é bastante) que a diagonal principal da matriz de Jacobi contenha as parcelas positivas (que descrevem a positiva conexão da volta).

O membro $a_{22}$ contém os elementos positivos $\alpha_{1} v_{\alpha}+\alpha_{2} \bar{X}$ que definem a causa do comportamento oscilatório na interação atrativa entre as partículas do filme. O primeiro elemento positivo define a interação durante a formação do filme e o segundo - durante a dissolução dela. Quanto mais a concentração dos íons de fluoreto (F), tanto o valor do segundo elemento (já que $\mathrm{X}>0$ ). Isto explica a dependência da freqüência, amplitude e período das oscilações observadas da concentração do fluoreto.

A instabilidade monotônica, cuja condição pode ser descrita como Det $\mathrm{J}=0$, para este sistema é também possível. A condição dela é

$$
\phi_{0}=\frac{D(X-1)\left(u+W_{2}\right)-S X F\left(a_{2}+1\right)+X F D\left(a_{2}-1\right)}{W_{1}(D X-\Delta D)}
$$

Neste caso no sistema existe a multidão dos estados estacionários entre os quais ele só escolhe um. Ele se destrói após mudança das condições do sistema. 


\section{CONCLUSÕES (SEÇÃO I)}

O sistema da oxidação dos íons de cobalto no eletrolito que contém fluoreto, foi descrito matematicamente. $\mathrm{O}$ modelo matemático correspondente foi analizado através da teoria da instabilidade linear e da análise de bifurcações.

$\mathrm{O}$ valor crítico do salto do potencial na camada dobrada elétrica, que definia a condição do estado estacionário estável foi encontrado usando o critério de Rauss e Gurwitz. A mudança do comportamento, observada experimentalmente foi explicada pela "entrada" do salto do potencial do anodo para os valores subcríticos. Os parâmetres que definiam a estabilidade do estado estacionário estável para este caso também foram achadas.

A causa do comportamento oscilatório neste sistema foi a interação atrativa entre as moléculas do filme. A influência da concentração dos íons de fluoreto também foi descrita.

A DESCRIÇÃO MATEMÁTICA DA SÍNTESE ELETROQUÍMICA DOS OXIHIDROXICOMPOSTOS DE COBALTO (III) NAS SOLUÇÕES SEM FLUORETO COM A ELETROPOLIMERIZAÇÃO SIMULTÂNEA DE UM COMPOSTO HETEROCÍCLICO.

Os polímeros condutores são uns dos compostos mais investigados durante as últimas décadas. Seu espectro de uso é muito vasto, começando nos revestimentos protetores de corrosão $[5,6] \mathrm{e}$ findando com os biossensores [7]. Também, como já foi dito, se usam os compósitos dos PC com as substâncias diferentes.

A reação da polimerização eletroquímica dos compostos heterocíclicos com aneis isolados $[6,8,9$, 10] e condensados [11] também foi observada a ser oscilatória. Foram observadas as oscilações em corrente $[10,11]$ no modo potenciostático e em potencial $[6,8$, 9] no modo galvanostático.

O objeto desta seção é o sistema da síntese eletroquímica do compósito do polímero condutor baseado em um composto heterocíclico com oxihidroxicomposto de cobalto na ausência dos ions de fluoreto.

Nós já fizemos a tentativa de descrever o sistema da imobilização das substâncias diferentes através da eletropolimerização dos compostos heterocíclicos [12]. No caso descrito em [12] a substância a se imobilizar entrava na matriz polimérica como dopante. Agora a oxidação dos íons de cobalto e a eletropolimerização oxidativa de um monômero heterocíclico, que possui o sistema conjugado, são duas reações paralelas.

Para este caso nós estamos introduzindo 3 variáveis

- c - a concentração do monômero na camada pre-superficial,

- $\theta$ - o grau do recobrimento da superfície do catodo pelo monômero

- $\sigma$ - a concentração dos cátions de cobalto na camada pre-superficial,

supondo o suposto acima.

O monômero na camada pre-superficial.O monômero entra na camada pre-superficial difundindo nela e desorvendo da superfície e sai dela adsorvendo. Então, a equação do balanço pode ser descrita como

$$
\frac{d c}{d t}=\frac{2}{\delta}\left(v_{-1}-v_{1}+\frac{D}{\delta}\left(c_{b}-c\right)\right) \equiv F_{1}
$$

Em que $v_{-1}$ e $v_{1}$ são velocidades de adsorção e desorção, D é o coeficiente da difusão do monômero, $\mathrm{c}_{\mathrm{b}}$ é a concentração do monômero por "dentro" da solução.

O monômero na superfície. O monômero chega pra superfície adsorvendo nela e sai dela desorvendo. Ele também se gasta polimerizando-se . A equação do balanço do monômero na superfície do catodo descrever-se-á assim

$$
\frac{d \Theta}{d t}=\Gamma_{t, \max }\left(v_{1}-v_{-1}-v_{2}\right) \equiv F_{2}
$$

Sendo $\tilde{A}_{t, \text { max }}$ a concentração maximal superficial do monômero $\mathrm{e} \mathrm{v}_{2}$ a velocidade da polimerização eletroredutiva dele.

Como na seção 1 , os cátions de cobalto entram na camada pre-superficial através da difusão e desaparecem oxidando-se eletroquimicamente. A equação de balanço então será descrita como

$$
\frac{d \sigma}{d t}=\frac{2}{\delta}\left(\frac{\Delta_{2}}{\delta}(\sigma-\Sigma)-v_{3}\right) \equiv F_{3}
$$


Sendo $\Sigma$ a concentração dos íons de cobalto no interior da solução, $\Delta_{2}$ o coeficiente da difusão dos cátions de cobalto, $\mathrm{v}_{3}$ a reação da oxidação.

$$
\begin{gathered}
v_{1}=k_{1} \exp \left(a_{2} \theta_{h}\right) c_{h}\left(1-\theta_{h}\right) \\
v_{2}=k_{2} \theta^{x} \exp \left(-\frac{z F}{R T} \phi_{0}\right) \\
v_{-1}=k_{-1} \exp \left(-a_{2} \theta_{h}\right) \theta_{h} \\
v_{3}=k_{o x} \sigma\left(1-\Theta_{h}\right) \exp \left(-\frac{z_{2} F}{R T} \phi_{0}\right)
\end{gathered}
$$

\section{RESULTADOS E DISCUSSÃO (SEÇÃO 2)}

Os elementos do jacobiano para o sistema de equações (15-17) para o estado estacionário serão mostrados como

$$
\begin{gathered}
a_{11}=\frac{\partial F_{1}}{\partial c}=\frac{2}{\delta}\left(-\frac{\partial v_{1}}{\partial c}-\frac{D}{\delta}\right) \quad a_{12}=\frac{\partial F_{1}}{\partial \theta}=\frac{2}{\delta}\left(-\frac{\partial v_{1}}{\partial \theta}+\frac{\partial v_{-1}}{\partial \theta}\right) \\
a_{12}=\frac{\partial F_{1}}{\partial \sigma}=0 \quad a_{21}=\frac{\partial F_{2}}{\partial c}=\Gamma_{\max 1}^{-1} \frac{\partial v_{1}}{\partial c} \\
a_{22}=\frac{\partial F_{2}}{\partial \theta_{n}}=\Gamma_{\max }^{-1}\left(\frac{\partial v_{1}}{\partial \theta}-\frac{\partial v_{-1}}{\partial \theta}-\frac{\partial v_{2}}{\partial \theta}\right) \quad a_{22}=\frac{\partial F_{2}}{\partial \theta_{h}}=0
\end{gathered}
$$

Analogicamente,

$$
\begin{gathered}
a_{31}=0 ; a_{31}=-\frac{v_{o x}}{1-\Theta}+\frac{z F}{R T} v_{o x} \frac{\phi_{0}\left(\mathrm{~K}_{0}-\mathrm{K}_{1}\right)+K_{1} \phi_{1}}{\mathrm{~K}_{1} \Theta+\mathrm{K}_{0}(1-\Theta)} \\
a_{33}=\frac{2}{\delta}\left(-\frac{\Delta_{2}}{\delta}-\frac{v_{o x}}{C}\right)
\end{gathered}
$$

Se, como acima, introduzir as variáveis

$$
\frac{\partial v_{1}}{\partial c}=\frac{v_{1}}{c}=w \frac{\partial v_{1}}{\partial \theta}=V ; \frac{\partial v_{-1}}{\partial \theta}=W ; \frac{\partial v_{2}}{\partial \theta}=X ; \frac{D}{\delta}=\kappa
$$

Sabendo que $\mathrm{a}_{33}$ é sempre negativo, podemos obter a condição do estado estacionário estável para este caso.

$$
\mathrm{X}(\mathrm{W}+\kappa)>0
$$

A primeira causa das oscilações neste sistema pode ser a interação atrativa entre as partículas adsortas. Isso depende as oscilações se as constantes $\alpha_{1}$ e $\alpha_{2}$. Neste caso as oscilações serão causadas pelo câmbio cíclico da resistência da superfície do anodo

A segunda causa das oscilações neste sistema A segunda causa das oscilações neste sistema são os câmbios da capacidade da camada dupla, causados pela redução catôdica dos oxidantes fortes a se formar durante a eletropolimerização. Isso depende o comportamento oscilatório no caso de. Isso vai ocorrer se $\varphi_{0}$ for negativo. A instabilidade monotônica (ou, seja a instabilidade da sela e nó) é também possível neste sistema sendo a condição dela

$$
\mathrm{X}(\mathrm{W}+\kappa)=0
$$

Então, pode-se concluír, que a oxidação dos sais de cobalto não é a causa das oscilações eletroquímicas. Elas sobrevêm por causa do afeto da eletropolimerização.

\section{CONCLUSÕES (SEÇÃO 2)}

1. O sistema da oxidação dos sais de cobalto (II) n com a simultânea eletropolimerização de um composto heterocíclico na ausência de ánion fluoreto foi descrita matematicamente. O modelo correspondente foi analizado através da teoria de instabilidade linear e análise de bifurcações.

2. É mostrado que a oxidação do cátion de cobalto não é responsável pelas oscilações ocorrentes neste sistema. O responsável por elas é o fator da eletropolimerização.

\section{REFERÊNCIAS}

1. Stadnik O. Diss. Kand. Chim. N. - Kiev. -2011

2. O. Stadnik et. al. - Int. Electrochem. Soc. Meeting. Abstract \# 2240. - 2010

3. M. Ogüz Güler et. al. -7 th Turkish Nanoscience and Nanotechnology Conference. - P. 24. - 2011

4. Roncali J.; Chem. Rev., 92(1992), 711

5. K.R. Lemos Castagno, Tés. D. Sc. UFRS.,Porto 
Alegre, 2007

6. M.S. Ba-Shmmakh. - Th. Deg. M.Sc. - King Saud Univ. Saudi Arabia. - 2003.

7. V.M. de Andrade, Tés. M. Eng. UFRS., Porto Alegre, 2006 e as referencias por dentro

8. I. Das, et. al // J. Phys. Chem. - 2009. -113. - P. $5296-5301$

9. I.Das, et. al // Indian Journal of Chemistry.2008.- V 47A.- pp.1798-1803

10. K.Aoki et. al // Russian Journal of Electrochemistry. - 2004. - 3. -P. 319 - 324

11. Sazou D.// Synthetic metals. - 2002. - V.130(1). - pp.45-54

12. V.V.Tkach, V.V. Nechyporuk// $4^{\text {th }}$ International Symposium on Flexible Organic Electronics. Thessaloniki. - 2011. - P. 146 